\title{
Symptom Experience and Quality of Life of Women Following Breast Cancer Treatment
}

\author{
NANCY K. JANZ, Ph.D., ${ }^{1}$ MAHASIN MUJAHID, Ph.D., ${ }^{2}$ LYNNA K. CHUNG, M.P.H., ${ }^{1}$ \\ PAULA M. LANTZ, Ph.D., ${ }^{3}$ SARAH T. HAWLEY, Ph.D., ${ }^{4,5}$ MONICA MORROW, M.D., ${ }^{6}$ \\ KENDRA SCHWARTZ, M.D., ${ }^{7}$ and STEVEN J. KATZ, M.D., M.P.H. ${ }^{3,4,5}$
}

\begin{abstract}
Background: Few studies have examined the correlates of breast cancer-related symptoms that persist posttreatment and determined the relationship between symptoms and quality of life (QOL).

Methods: A population-based sample of women in the United States with stage 0-II breast cancer $(n=1372)$ completed a survey including the European Organization for Research and Treatment of Cancer Quality of Life Questionnaire and the Breast Cancer-Specific Quality of Life Questionnaire. Described are the presence and frequency of 13 symptom scales and their associations with 10 QOL dimensions.

Results: All study participants had completed primary treatment (surgery and radiation and/or chemotherapy, if applicable). Mean time from initial surgical treatment to completion of the questionnaire was 7.2 months (range 0.5-14.9 months). Mean number of symptoms reported was 6.8, with the 5 most common symptom scales being systemic therapy side effects $(87.7 \%)$, fatigue $(81.7 \%)$, breast symptoms $(72.1 \%)$, sleep disturbance $(57.1 \%)$, and arm symptoms $(55.6 \%)$. Younger age and poorer health status at diagnosis were associated with worse symptoms. Fatigue had the greatest impact on QOL, with significant differences between those with high and low fatigue across 7 QOL dimensions. Sociodemographic, prior health status, clinical, and treatment/diagnostic factors explained only $9 \%-27 \%$ of the variance in QOL outcomes. Adding symptom experience increased the variance explained to $18 \%-60 \%$.

Conclusions: More attention to the reduction and management of disease and treatment-related symptoms could improve QOL among women with breast cancer.
\end{abstract}

\footnotetext{
${ }^{1}$ Department of Health Behavior and Health Education, ${ }^{2}$ Department of Epidemiology, and ${ }^{3}$ Department of Health Management and Policy, University of Michigan, School of Public Health, Ann Arbor, Michigan.

${ }^{4}$ Division of General Medicine, Department of Internal Medicine, University of Michigan Health System, Ann Arbor, Michigan.

${ }^{5}$ Veterans Affairs Ann Arbor Healthcare System, Ann Arbor, Michigan.

${ }^{6}$ Department of Surgical Oncology, Fox Chase Cancer Center, Philadelphia, Pennsylvania.

${ }^{7}$ Department of Family Medicine and Karmanos Cancer Institute, Wayne State University, Detroit, Michigan.

This work was funded by a grant from the National Cancer Institute (R01 CA8837-A1) to the University of Michigan. This project has been funded in part with federal funds from the National Cancer Institute, National Institutes of Health, Department of Health and Human Services, under Contract No. N01-PC-35139 and N01-PC-65064. The collection of cancer incidence data used in this publication was supported by the California Department of Health Services as part of the statewide cancer reporting program mandated by California Health and Safety Code Section 103885.

The ideas and opinions expressed herein are those of the authors, and no endorsement by the State of California Department of Health Services is intended or should be inferred.
} 


\section{INTRODUCTION}

$\mathbf{W}$ WOMEN WTH A DIAGNOSOS OF BBEAST CANCER represent one of the largest groups of cancer survivors, comprising about $22 \%$ of the nearly 10 million cancer survivors in the United States. ${ }^{1}$ In fact, one of the priorities highlighted in the National Cancer Institute's 2003-2004 Annual Report on Cancer Survivorship was that "the transition from active treatment to social reintegration is crucial and should receive specific attention in survivors' care." ${ }^{2}$ Although the majority of the literature has focused on treatment and supportive care provided to women during the initial treatment phase, far less attention has been given to improving recovery and quality of life (QOL) on completion of primary treatment. Evidence suggests that even after primary treatment women continue to experience QOL concerns, including emotional distress, fear of recurrence, and difficulties resuming family, work, and social roles. ${ }^{3}$ Women who are highly distressed during early survivorship report poorer long-term adjustment outcomes. ${ }^{4}$

Studies have found that many symptoms persist after completion of primary breast cancer treatment, including problems with fatigue, ${ }^{5,6}$ pain, ${ }^{7}$ lymphedema, ${ }^{8-10}$ menopausal symptoms, ${ }^{11-15}$ and sleep disturbance. ${ }^{16-18}$ In particular, fatigue has been reported by women irrespective of treatment course. ${ }^{5,6,19}$ Most studies have focused on individual symptoms or a unique subset of symptoms (i.e., menopausal) rather than examining the relative impact of a woman's collective symptom experience on QOL.

Examining the correlates of symptom experience on population subgroups has usually involved a limited number of sociodemographic characteristics or various aspects of the treatment course. Some studies have suggested that symptom experience differs by age, $, 6,20-22$ education, $, 21,23,24$ income, ${ }^{25}$ and race/ethnicity. ${ }^{25}$ Symptom experience has also been purported to vary by type of treatment, although the evidence is mixed. $21,23,26,27$ Whereas some researchers have found very little variation in physical symptoms and side effects across some combinations of treatment, for example, surgery (lumpectomy or mastectomy), chemotherapy, and radiation therapy, ${ }^{23,27}$ others have found that symptom reporting varies by treatment modality and over time. ${ }^{21,26}$

Few studies have examined the association of women's symptom experience with their QOL as- sessment. Ganz et al. ${ }^{26}$ explored the extent to which symptoms contribute to the assessment of physical and mental well-being among 558 women completing primary treatment. The authors concluded that such symptoms as muscle stiffness, breast sensitivity, aches and pains, tendency to take naps, and difficulty concentrating were common among women finishing primary treatment and were statistically significantly associated with poor physical and emotional well-being. Arndt et al. ${ }^{28}$ targeted women in early survivorship using a comprehensive cancer-specific measure of symptoms and QOL to assess the relative impact of symptom experience across QOL domains. The study measured the relationship between symptom experience and QOL among 314 women in Germany 1 year after primary treatment for breast cancer. Fatigue was found to be the strongest predictor of QOL.

Gaining a better understanding of the symptom experience and its impact on QOL may inform intervention strategies to assist women in this vulnerable stage of breast cancer survivorship. The objectives for this research study were (1) to describe the symptom experience of women following primary breast cancer treatment, (2) to determine the correlates of breast cancer symptoms, and (3) to examine the relationship of symptom reporting to various dimensions of QOL.

\section{MATERIALS AND METHODS}

\section{Study population}

The study population consisted of women diagnosed with ductal carcinoma in situ (DCIS) and invasive, nonmetastatic breast cancer in the greater metropolitan areas of Detroit, Michigan, and Los Angeles, California. Women were identified using the Surveillance, Epidemiology, and End Results (SEER) registries in Detroit and Los Angeles between December 2001 and January 2003. All cases of DCIS and an approximate $20 \%$ random sample of invasive cases were selected in both cities. Eligibility criteria included (1) age $\leq$ 79 years, (2) a primary diagnosis of breast cancer treated with a definitive surgical procedure, and (3) ability to complete a written or telephone survey in English or Spanish. African American women with invasive disease were oversampled to increase their representation to approximately 
one third in Detroit and 20\% in Los Angeles. Because Asian women in Los Angeles were being approached for other studies during the project period, they were not recruited for this study. Women were also excluded if they had a previous history of primary breast cancer, had metastatic disease, or had a diagnosis of lobular carcinoma in situ (CIS). For the purposes of this study, we also excluded women who had not completed their primary treatment course for breast cancer (i.e., surgery and radiation and/or chemotherapy, if applicable).

Once SEER staff members identified potential participants, the following protocol was initiated. Physicians were notified of our intent to contact patients $(<1 \%$ were excluded due to physician concerns), and an introductory letter was sent to potential participants, followed by a phone call to assess eligibility. A questionnaire and a $\$ 10$ coupon for a local grocery store were mailed to all who agreed to participate and met eligibility criteria. The Dillman method was used to encourage response rates, ${ }^{29}$ involving a postcard reminder for nonrespondents at 2 weeks, a second letter and survey at 6 weeks, and a follow-up phone call at 10 weeks. At the follow-up phone call, nonrespondents were offered, if they preferred, a short telephone survey.

After removing personal identifiers, the SEER sites sent completed surveys to the research team at the University of Michigan for data entry and analysis. Subsequently, SEER pathology and survey data were merged using a unique patient identification number. The study protocol was approved by the Institutional Review Boards of the University of Michigan, Wayne State University, and the University of Southern California.

\section{Study measures}

Symptom experience and health-related QOL. Symptom experience and QOL were assessed using the European Organization for Research and Treatment of Cancer Quality of Life Questionnaire (EORTC QLQ-C30) ${ }^{30}$ and the Breast Cancer-Specific Quality of Life Questionnaire (QLQBR23). ${ }^{31}$ The EORTC QLQ-C30 version 2 consists of 30 questions addressing five functional domains (physical, emotional, role, social, cognitive), one global QOL domain, three symptom domains (fatigue, pain, nausea/vomiting), and six single items (sleep disturbance, dyspnea, appetite loss, constipation, diarrhea, financial impact). The breast cancer-specific supplement (QLQ-BR23) consists of 23 items, including two functional domains (body image, sexual functioning), three symptom domains (breast symptoms, arm symptoms, systemic therapy side effects), and three single items (future perspective, sexual enjoyment, upset by hair loss). Respondents were asked to report if they had experienced symptoms, using the past week as a time frame, with possible responses as yes / no or a 4-point scale (1, not at all, to 4, very much), except for items related to sexual functioning and enjoyment, where the time frame was during the past 4 weeks. For all items within the EORTC QLQ-C30 and the QLQ-BR23, higher scores indicate better QOL (i.e., better functioning) but worse symptom experience. The psychometric properties of the EORTC and QLQ-BR23 have been well established with excellent reliability and validity. Both instruments have been used with breast cancer patients. $^{32,33}$

A major advantage of the EORTC QLQ-C30 is the considerable work that has been published on interpretation of clinically relevant difference scores. ${ }^{34,35}$ Osoba et al. ${ }^{35}$ found that patients with breast and lung cancer who reported a small change or a moderate change in symptom experience over time also reported a corresponding mean change of 5-9 points or 10-20 points on their QLQ-C30 scores, respectively. Based on these results, we set the criterion of a betweengroup difference of 10 or more points to represent a clinically meaningful difference.

Additional variables. Information on sociodemographic characteristics, prior health status, clinical factors, and breast cancer treatment/diagnostic factors were also obtained from survey data.

Sociodemographic characteristics of interest included age at the time of breast cancer diagnosis, race, education level, total yearly household income, and marital status.

Prior health status variables included the selfreported number of comorbid conditions (chronic bronchitis, heart disease, diabetes, high blood pressure, stroke, arthritis) and health status at time of diagnosis rated on a 5-point scale (poor, fair, good, very good, excellent).

Clinical factors included breast cancer stage using American Joint Committee on Cancer criteria, ${ }^{36}$ and the time interval between primary breast cancer surgery and completion of the survey. 
Treatment/diagnostic factors included initial surgical treatment (mastectomy with and without reconstruction vs. breast-conserving surgery [BCS]), whether or not axillary node dissection was done, and receipt of chemotherapy, radiation therapy, and/or hormone therapy. SEER summary information was used to determine if a woman had axillary node dissection as well as to fill in missing data from participant self-report for the following measures: age at diagnosis, race, marital status, response time from date of diagnosis to survey completion, primary surgical treatment, chemotherapy, and radiation therapy.

\section{Statistical methods}

The data were weighted to account for the sampling design and to correct for nonresponse. To account for those women who could not be reached by mail or telephone, we used a weight of 0.9 to reflect imputed eligibility if we had made contact. Descriptive statistics were performed to characterize the distribution of the variables for the sample. In addition, in order to describe the symptom experience of women with breast cancer, we constructed indicator variables for each symptom item within the 13 symptom scales. We also constructed an indicator variable for each of the 13 symptom scales, defined as endorsing at least one item within the set of items representing the scale.

Using a linear transformation as instructed by the EORTC QLQ scoring manual, we created 10 QOL scales (physical, emotional, social, role, and cognitive functioning, global QOL, future perspective, body image, sexual functioning, sexual enjoyment). Additionally, we created a scale for the 13 symptom domains, of which 6 are multiitem scales (fatigue, pain, nausea/vomiting, systemic therapy side effects, breast symptoms, arm symptoms) and the remaining are single item scales (sleep disturbance, dyspnea, appetite loss, constipation, diarrhea, financial impact, upset by hair loss). All scales (QOL, multi/single item symptom scales) ranged from 0 to 100 , with higher scores indicating better QOL and worse symptom experience.

Analysis of covariance (ANCOVA) was used to test for associations between symptom scales and sociodemographic, prior health status, clinical, and treatment/diagnostic factors, as well as for associations between symptom scales and QOL outcomes. Only scales that were endorsed by at least $50 \%$ of our analytical sample were included in these analyses. We also calculated estimates of the mean difference in QOL between the 90th (high symptom experience) and 10th (low symptom experience) percentile of each symptom scale after adjusting for sociodemographic, prior health status, clinical, and treatment/diagnostic factors. We considered between-group differences that were statistically significant and 10 or more points to be clinically meaningful differences. Because all hypotheses were specified $a$ priori, no adjustments were made for multiple comparisons. However, $\mathrm{p}<0.001$ can be viewed as statistically significant by the most conservative methods of adjustment for multiple comparisons. All analyses were performed using SAS version 8.2 (SAS Institute, Cary, NC).

\section{RESULTS}

Of the 2640 cases identified through the SEER registries, $88 \%(n=2323)$ were eligible, and $74.3 \%$ completed the survey $(77.0 \%$ from Los Angeles and $77.9 \%$ from Detroit). The percent of women who were ineligible due to death/illness, no definitive surgical procedure at contact, or language barrier were similar across sites. Among those women who completed the survey, 93\% completed the written survey, and $7 \%$ completed an abbreviated telephone survey. Nonrespondents were more likely to be nonwhite and older and have advanced disease. Additional exclusions from the 1844 participants in the final study were made based on the specific aims of this substudy, including 141 women who had completed the telephone survey where QOL measures were not available, 148 women with late stage disease (stage III), and 183 women who had not completed their primary treatment course (i.e., surgery and radiation and/or chemotherapy). A total of 1372 women were retained for our final analytical sample.

Table 1 summarizes the sociodemographic and treatment characteristics of our study sample. The cutoff points for age were $<45$ years, $45-54$ years, 55-64 years, and $\geq 65$ years of age. About $37 \%$ of women were $\geq 65$ years of age, and almost $9 \%$ were $<45$ years of age. The racial breakdown was $70.4 \%$ non-Hispanic white, $15.2 \%$ non-Hispanic African American, and $14.4 \%$ other, of which $85 \%$ were Hispanic. The majority of our sample was married or with a domestic partner 
Table 1. Characteristics of Women in Study $(N=1372)^{\mathrm{a}}$

\begin{tabular}{|c|c|c|c|}
\hline Characteristics & n (\% weighted) & Characteristics & $\mathrm{n}(\%$ weighted $)$ \\
\hline Sociodemographics & & Clinical factors & \\
\hline Study site & & Cancer stage & \\
\hline Los Angeles & $729(52.4)$ & 0 & $598(25.7)$ \\
\hline Detroit & $643(47.6)$ & I & $482(48.0)$ \\
\hline \multicolumn{4}{|l|}{ Age } \\
\hline Mean age, years $(S D)^{b}$ & $60.5(10.7)$ & II & $253(26.3)$ \\
\hline$<45$ & $141(8.9)$ & Mean time from initial surgery to & $7.2(2.5)$ \\
\hline $45-54$ & $330(21.9)$ & completion of questionnaire, & \\
\hline $55-64$ & $420(32.1)$ & months $(\mathrm{SD})^{\mathrm{b}}$ & \\
\hline $65+$ & $481(37.1)$ & Treatment/diagnostic factors & \\
\hline Race & & Primary surgical treatment & \\
\hline White, (non-Hispanic) & $917(70.4)$ & Breast-conserving surgery & $942(70.1)$ \\
\hline African American, (non-Hispanic) & $258(15.2)$ & Mastectomy & $430(29.9)$ \\
\hline Other & $191(14.4)$ & Axillary node dissection & \\
\hline Education & & Yes & $779(61.8)$ \\
\hline Less than high school & $174(13.1)$ & No & $593(38.2)$ \\
\hline High school diploma & $299(22.7)$ & Radiation therapyc & \\
\hline Some college or beyond & $885(64.2)$ & Yes & $828(73.8)$ \\
\hline Income & & No & $544(26.2)$ \\
\hline$<\$ 25,000$ & $275(19.7)$ & Chemotherapy ${ }^{c}$ & \\
\hline$\$ 25,000-\$ 49,999$ & $383(28.1)$ & Yes & $276(27.3)$ \\
\hline$\geq \$ 50,000$ & $560(41.0)$ & No & $1091(72.7)$ \\
\hline Unknown & $154(11.2)$ & Hormone therapy ${ }^{\mathrm{d}}$ & \\
\hline Marital status & & Yes & $698(55.9)$ \\
\hline Currently married/domestic partner & $815(60.1)$ & No & $662(44.1)$ \\
\hline Divorced/separated/widowed & $449(33.0)$ & & \\
\hline Never married & $108(6.9)$ & & \\
\hline \multicolumn{4}{|l|}{ Prior health status } \\
\hline \multicolumn{4}{|l|}{ Number of comorbidities } \\
\hline 0 & $539(39.6)$ & & \\
\hline 1 & $399(29.1)$ & & \\
\hline $2+$ & $431(31.3)$ & & \\
\hline \multicolumn{4}{|l|}{ Health status at diagnosis } \\
\hline Poor/fair & $170(12.2)$ & & \\
\hline Good & $449(33.5)$ & & \\
\hline Very good/excellent & $734(54.3)$ & & \\
\hline
\end{tabular}

aSample size is unweighted, percents are weighted; less than 3\% missing data across all variables.

bSD, standard deviation.

'For radiation therapy and chemotherapy, Yes indicates number of women who had completed treatment.

dFor hormone therapy, Yes indicates number of women who had started or finished treatment.

and with some college education. Approximately $41 \%$ of women reported an income of at least $\$ 50,000,28 \%$ reported an income between $\$ 25,000$ and $\$ 49,999$, and $20 \%$ reported an income $<\$ 25,000$. About two thirds of the women reported fewer than two comorbid conditions, and a little over half reported very good/excellent health status at time of diagnosis. About $26 \%$ of women had stage $0,48 \%$ stage I, and $26 \%$ stage II breast cancer. The mean time from initial surgery was 7.2 months (range $0.5-14.9$ months). Approximately $70 \%$ of women received BCS, and $30 \%$ had a mastectomy. Of those who had a mastectomy, $42.8 \%$ had reconstruction. About $74 \%$ of women in the sample had axillary node dissec- tion. All women had completed primary treatment, $62 \%$ had completed radiation (most of these women had BCS), and $27 \%$ had completed chemotherapy. At the time women filled out the questionnaire, $56 \%$ reported being on hormone therapy.

Table 2 summarizes the means and standard deviations (SD) of all symptom and QOL scales. Cronbach's alphas for the QOL dimensions and multi-item symptom scales were primarily $>0.60$ (physical functioning 0.65; emotional 0.88; role 0.87; social 0.84; cognitive 0.69; body image 0.88; sexual functioning 0.79; global health 0.92; fatigue 0.84 ; pain 0.80 ; systemic therapy side effects 0.41 ; nausea/vomiting 0.63 ; breast symptoms 0.77 ; 
Table 2. Mean (SD) of Symptom Scales and Quality of Life Dimensions ${ }^{\mathrm{a}}$

\begin{tabular}{|c|c|c|c|}
\hline Symptom scales ${ }^{\mathrm{b}}$ & Mean (SD) & Qualify of Life Dimensions ${ }^{c}$ & Mean (SD) \\
\hline \multicolumn{4}{|l|}{ EORTC QLQ-C30 } \\
\hline Fatigue & $28.71(22.4)$ & Physical functioning & $82.57(21.6)$ \\
\hline Sleep disturbance & $27.35(27.9)$ & Emotional functioning & $75.52(21.4)$ \\
\hline Pain & $17.90(22.9)$ & Role functioning & $82.59(24.4)$ \\
\hline Dyspnea & $14.20(23.0)$ & Social functioning & $86.57(21.2)$ \\
\hline Constipation & $13.22(22.2)$ & Cognitive functioning & $82.51(20.8)$ \\
\hline Appetite loss & 8.77 (18.4) & Global quality of life & $71.40(20.4)$ \\
\hline Nausea and vomiting & $4.35(12.0)$ & & \\
\hline Diarrhea & $6.23(15.6)$ & & \\
\hline Financial impact & $17.90(27.6)$ & & \\
\hline \multicolumn{4}{|l|}{ EORTC QLQ-BR23 } \\
\hline Systemic therapy side effects & $18.47(15.3)$ & Future perspective & $62.14(29.1)$ \\
\hline Breast symptoms & $18.23(18.5)$ & Body image & $84.60(21.8)$ \\
\hline Arm symptoms & $15.15(19.5)$ & Sexual functioning & $20.86(21.4)$ \\
\hline Upset by hair loss & $14.43(29.2)$ & Sexual enjoyment ${ }^{\mathrm{d}}$ & $51.64(29.1)$ \\
\hline
\end{tabular}

aAll symptom scales and quality of life dimensions scored from 0 to 100 .

${ }^{b}$ Higher scores for symptom scales indicate worse symptom experience.

'Higher scores for quality of life dimensions indicate better quality of life.

dOnly includes women who were sexually active $(n=641)$.

arm symptom 0.74$)$. Scale scores incorporate not only the presence or absence of the symptom but also the frequency, a further indication of impact on QOL. Among the scales, women reported the highest scores (worse symptom experience) for fatigue and sleep disturbance and the lowest mean QOL scores for sexual functioning, sexual enjoyment, and future perspective.

The individual symptoms reported by women are summarized in Figure 1. Identified at the bottom of Figure 1 are the individual items with their associated symptom scales. Among the individual symptoms, feeling tired was endorsed by the largest proportion of women $(75.5 \%)$, followed by needing rest $(66.2 \%)$, hot flashes $(62.3 \%)$, sleep disturbance $(57.1 \%)$, and pain in the area of the breast $(52.5 \%)$. Far fewer women reported experiencing swollen arms, nausea/vomiting, or diarrhea. By creating an indicator variable for each symptom scale, we found that $>50 \%$ of women reported experiencing at least one symptom within the following scales: systemic therapy side effects $(87.7 \%)$, fatigue $(81.7 \%)$, breast symptoms (72.1\%), arm symptoms (55.6\%), and pain (51.7\%) (data not shown). The mean number of symptom scales endorsed was 6.18 (range 1-13), and $<5 \%$ of women reported less than three symptoms.

In an effort to focus on the symptoms that were most often reported by women, all further analyses were restricted to the 6 symptom scales reported by at least $50 \%$ of the sample. Table 3 sum- marizes the statistically significant and clinically meaningful results from ANCOVA models regressing each of these symptom scales on sociodemographic, prior health status, clinical, and treatment/diagnostic factors. Only the clinically meaningful results are highlighted. With respect to sociodemographic factors, clinically meaningful differences ( $\geq 10$ point difference) were only present for age, with younger women $(<45$ years of age) experiencing worse fatigue, pain, and breast symptoms than those women $\geq 65$ years of age. Health status at diagnosis was the measure most consistently associated with breast cancer symptom experience, with women who reported good or very good/excellent (compared with those who reported fair/poor) health status at diagnosis having better symptom experience related to fatigue, sleep disturbance, and pain. With regard to cancer stage, women with more advanced disease at diagnosis reported less sleep disturbance. Treatment/diagnostic differences in symptom experience were primarily a function of chemotherapy, with women who had chemotherapy having more problems with sleep. There were no clinically meaningful differences in symptom experience between women who received mastectomy and those who received BCS. Together, these sociodemographic, prior health status, clinical, and treatment/diagnostic factors explained between $7 \%$ and $17 \%$ of the variability in symptom experience. 


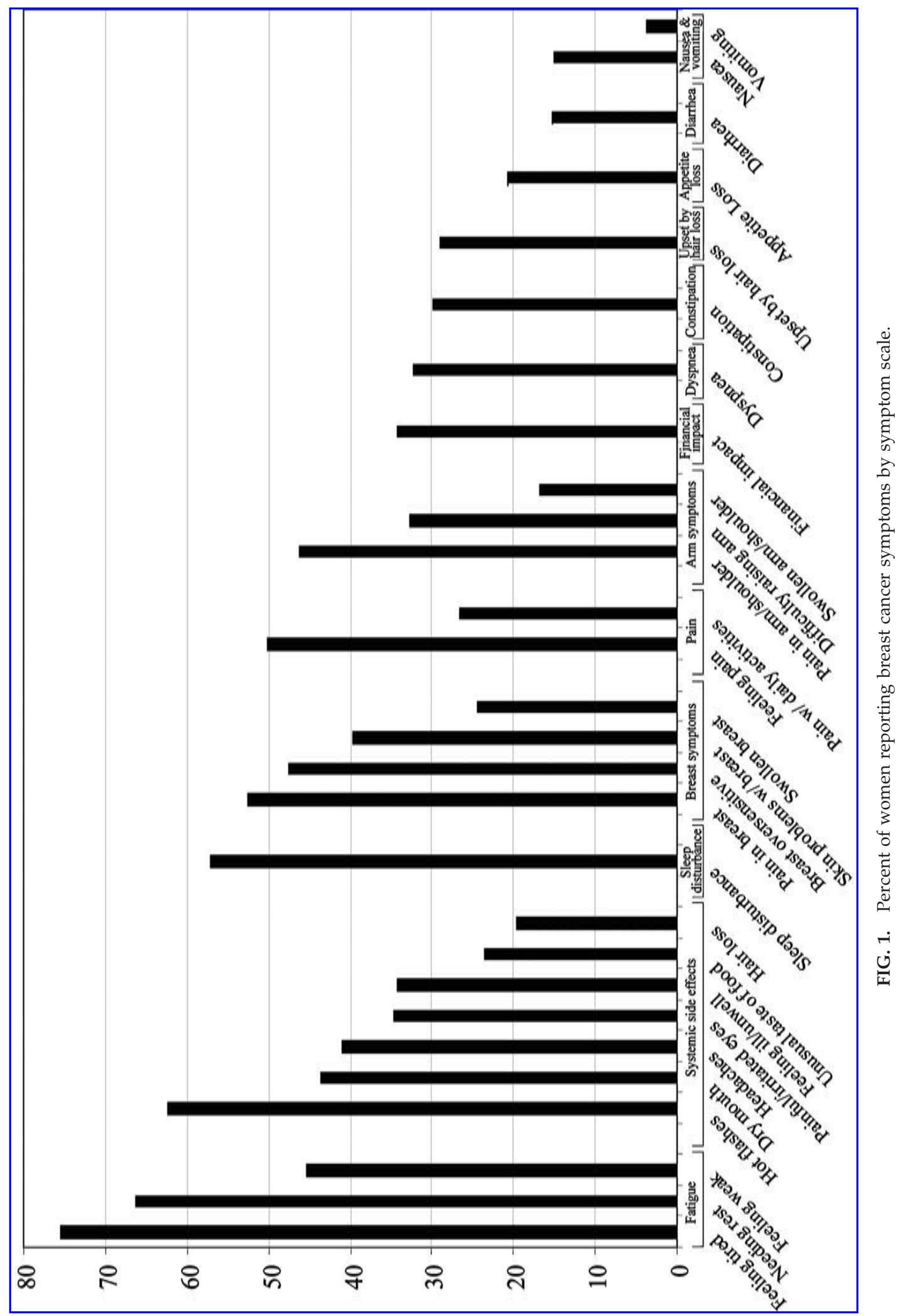


Table 3. Results of Multiple Linear Regression Models of Symptoms Scales and Sociodemographic, Prior Health Status, Clinical, and Treatment/Diagnostic Factors ${ }^{a}$

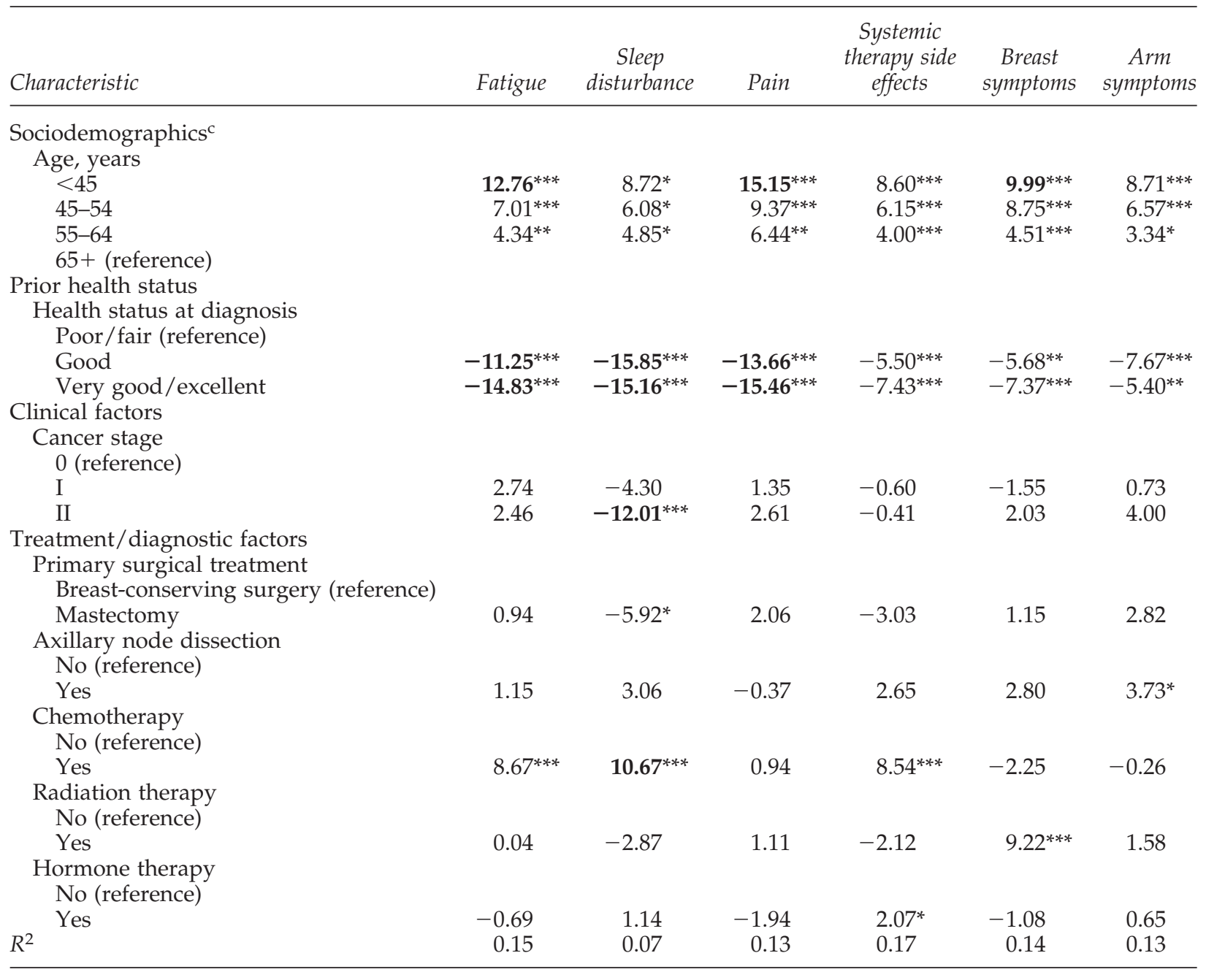

aAll symptom scales scored from 0 to 100 .

bValues represent mean differences in QOL outcomes between high (90th percentile) and low (10th percentile, reference). Values in bold indicate clinically meaningful findings, as defined by a $\geq 10$ difference on a scale from 0 to 100. Model also adjusts for study site, race, education, income, marital status, cancer stage, axillary node dissection, number of comorbidities, and time from treatment.

'Model results for race, education, income, and marital status are not reported in the table, as there were no clinically meaningful findings.

${ }^{*} p<0.05$.

$* * p<0.01$

$* * * p<0.001$.

Table 4 shows the mean differences in QOL outcomes between the 90th (high symptom experience) and the 10th (low symptom experience) percentiles of each symptom scale after adjusting for sociodemographic, prior health status, clinical, and treatment/diagnostic factors and other symptoms scales. In general, higher symptom scale scores were associated with decreased QOL outcomes. Fatigue had the most impact on QOL outcomes, with clinically meaningful differences between the 90th and 10th percentiles for 7 QOL dimensions (all differences $>15$ points). For example, for role functioning the average score for women with high fatigue (90th percentile) was 27.84 points lower than that of women with low fatigue (10th percentile). Other clinically meaningful differences were evident with respect to sleep disturbance (for emotional functioning), pain (for physical, social, and role functioning), systemic therapy side effects (for cognitive func- 


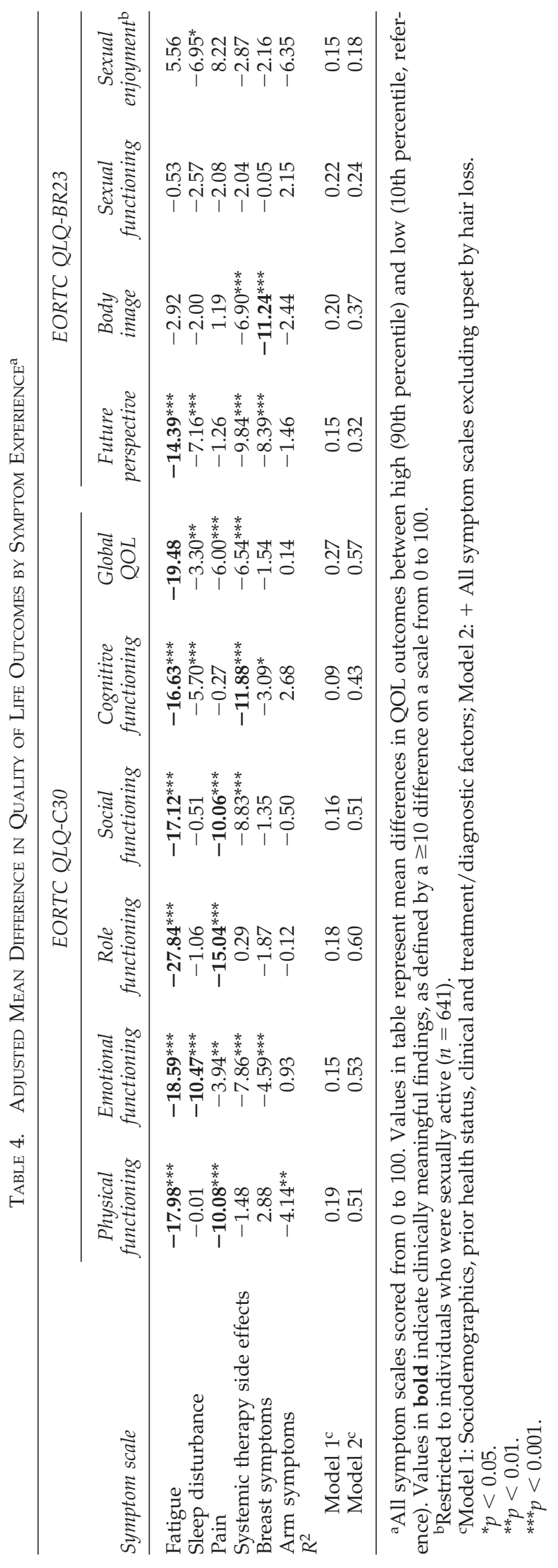


tioning), and breast symptoms (for body image). Table 4 also compares the percent of the variability in our QOL dimensions explained by all sociodemographic, prior health status, clinical, and treatment/diagnostic factors with and without symptom experience in the model. The sociodemographic, prior health status, clinical, and treatment/diagnostic factors explained only $9 \%-27 \%$ of the variability in QOL outcomes. The addition of symptom experience markedly increased the variance explained to between $18 \%$ and $60 \%$. For example, the variance explained in role functioning increased from 0.18 to 0.60 when symptom experience was added to the model. The only two outcomes where adding symptom experience produced only minor increases in variance explained were sexual functioning and sexual enjoyment.

\section{DISCUSSION}

The findings from this large population-based study provide further insight into the symptom experience and its impact on QOL among women who have completed primary treatment for breast cancer. During the vulnerable period of transitioning to survivorship, it is apparent that women continue to experience multiple symptoms. ${ }^{26}$ Over half of the women in our study reported symptoms related to fatigue, hot flashes, sleep disturbance, general pain, and breast discomfort. In another multisite study, Ganz et al. ${ }^{26}$ reported similar findings on the symptom experience of women at the end of primary treatment: $60 \%$ reported aches and pains, $60 \%$ reported hot flashes, and $56 \%$ reported breast sensitivity. In general, sleep disturbance has been noted in cancer patients ${ }^{17}$ but seems to be particularly problematic for breast cancer patients, with 38\%-61\% reporting sleep difficulties. ${ }^{16}$ Findings from other studies show variation in the persistence of these symptoms. King et al. ${ }^{21}$ found that women reported significant improvements in fatigue, pain, and chest and breast symptoms between 3 months and 1 year after breast cancer surgery. However, Hartl et al. ${ }^{37}$ surveyed women with breast cancer an average of 4.2 years after treatment using the EORTC QLQ-C30 and found that the most frequent complaints continued to be sleep disturbance, fatigue, and pain.

Our findings suggest that sociodemographic characteristics generally do not help define wo- men who will have greater or lesser symptom experience following breast cancer treatment. The only clinically meaningful exception was present when comparing younger and older women. Independent of other baseline health status and treatment-related factors, younger women had worse symptom experience. The finding that breast cancer has a disproportionate negative impact on younger women ( $<45$ years of age) has been reported by others. ${ }^{20,21,38}$ Although we controlled for treatment in our analyses, approximately $46 \%$ of women $<45$ years of age were treated with chemotherapy. Menopausal transition for younger women has been associated with decreases in QOL. ${ }^{20}$ Previous studies have offered potential explanations for why younger women may report greater declines in QOL. These include that younger women may feel more vulnerable, ${ }^{20,39}$ experience more emotional distress, ${ }^{20}$ have greater fear of death,${ }^{40}$ have more disruptions in their daily activities and finances, ${ }^{39}$ and possess fewer coping strategies. ${ }^{20}$

Results from this study lend support to research suggesting that sociodemographic, prior health status, clinical, and treatment/diagnostic factors explain only a modest amount of the variance in QOL among women following completion of primary treatment. ${ }^{28,41}$ Additionally, we found that women's symptom experience had a significant impact across a number of dimensions of QOL. In particular, higher levels of fatigue were associated with substantial reduction across multiple domains of QOL. The important contribution of fatigue to QOL has been documented previously. Baron et al. ${ }^{42}$ found that persistent fatigue following breast cancer treatment interfered with functioning and had a negative effect on physical, mental, and psychological well-being. A recent study reported that after adjusting for age, severity of fatigue explained approximately $30 \%-50 \%$ of the variability across functional areas. ${ }^{28}$ In addition, Bower et al. ${ }^{6}$ reported that approximately one third of breast cancer survivors had severe fatigue, which was associated with significantly higher levels of depression, pain, and sleep disturbance.

Other symptoms that contributed substantially to QOL after primary treatment included sleep disturbance, pain, and systemic therapy side effects. In addition to fatigue, Arndt et al. ${ }^{28}$ found that pain, systemic therapy side effects, and arm symptoms were the symptoms most highly correlated with QOL. Others have reported that 
breast cancer patients with significant sleep problems have reduced ability to perform work and accomplish physical tasks. ${ }^{18}$ Although we did not find a clinically important relationship between pain and type of surgical treatment, others have suggested that the pain secondary to surgery or radiation can be a strong predictor of fatigue ${ }^{6}$ and, if it persists, can cause considerable disability and psychological distress. ${ }^{43}$ Finally, our results on the impact of symptoms on QOL are consistent with those of a study conducted by Ganz et al., ${ }^{26}$ which concluded that the severity of symptoms experienced by women after primary treatment was significantly related to physical and mental well-being.

Several previous studies have noted that women report difficulties with sexual functioning and sexual enjoyment following completion of primary treatment for breast cancer. ${ }^{44,45}$ Our results suggest that symptoms do not play a major role in understanding changes in sexual functioning. It is important to note that some symptoms, such as vaginal dryness, were not measured in this study. Previous studies have reported that lack of desire, greater body image problems, difficulty with arousal, and partnerrelated issues are important contributors. ${ }^{39,45} \mathrm{In}$ a recent review of the literature for breast cancer survivors 5 years or more postdiagnosis, Mols et al. ${ }^{46}$ concluded that although most women reported good QOL, one of the problems that could persist was difficulties with sexual functioning.

\section{Limitations}

Our study findings are limited by the cross-sectional design and absence of baseline symptom and QOL assessment prior to breast cancer diagnosis. Therefore, one has to interpret any attribution of symptoms to breast cancer and its treatment with some caution. A previous study by Schou et al. ${ }^{47}$ that compared women with breast cancer to a general female population found that some of these symptoms are common to women in this age group. In addition, the generalizability of our findings to nonwhite racial/ethnic groups is somewhat compromised by the practical limitation imposed by excluding Asian women at one site and the relatively small sample of racial/ethnic groups outside of African Americans. Although we measured a number of comorbidities and health status at diagnosis and controlled for them in the analyses, we may have attributed some symptom reporting to breast cancer and its treatment that is actually the result of other conditions, such as age-related menopause. Finally, some may question the definition of clinically meaningful differences in this study, which was based on previous work by Osoba et al. ${ }^{35}$ However, we believe it is important to avoid the pitfall of a large sample size yielding statistically significant findings that are not likely to be clinically meaningful.

\section{CONCLUSIONS}

The findings of this study have implications for clinical practice and future research. The fact that breast cancer symptoms accounted for a significant amount of the variability in QOL dimensions suggests that reducing the symptom burden should have a positive effect on QOL. Upon completion of primary treatment, women experience reduced contact with healthcare providers at a time when they are still in need of support and are at risk for adjustment difficulties. ${ }^{26}$ Systematic documentation of the presence and severity of symptoms at the end of primary treatment would be a reasonable first step. At present, follow-up visits are often focused on detecting symptoms of recurrence even though recurrence is uncommon in the early posttreatment period. For some women, acknowledgment of their symptoms, counseling about common posttreatment symptoms and their natural history, or teaching behavioral self-management skills to deal with symptoms that persist may be all that is required. An assessment to determine if symptoms are worsening, stable, improving, or resolved seems warranted by the findings of our study.

For women experiencing more significant symptoms at the completion of primary treatment, further therapy to control persistent symptoms may be required. For example, pain and stiffness as a result of axillary surgery can be improved with physical therapy, and hot flashes can be reduced with low-dose antidepressants. ${ }^{48}$ Fatigue has multiple potential etiologies, but persistent severe fatigue should prompt an evaluation for anemia and depression. Treating anemia-related fatigue with hemoglobin has been found not only to improve fatigue but also to lead to improvements in physical and emotional well-being. ${ }^{49}$ 
Our findings suggest that assessment and interventions aimed at reducing fatigue, sleep disturbance, and pain should target those patients in whom these symptoms are most prevalentyounger women and those with a worse health status at the time of diagnosis. The fact that sociodemographic factors generally were not associated with symptom scores or QOL dimensions suggests that assessment and interventions should be broadly considered across these subgroups. Bower et al. ${ }^{6}$ suggested that focusing efforts on treating symptoms of depression, pain, and sleep disturbance should prove useful in combating fatigue. The negative association between fatigue and QOL highlights the importance of developing and evaluating behavioral interventions to treat women with considerable fatigue. Cimprich and Ronis ${ }^{50,51}$ found an intervention focused on mental restoration and involving regular exposure to natural environments was beneficial in counteracting fatigue and significantly improving cognitive function in women treated for breast cancer. In addition, difficulties in sexual functioning and enjoyment seem to involve a more complicated set of factors beyond just medical and treatment characteristics, requiring further understanding, investigation, and intervention.

Early assessment and treatment of symptoms have the potential to reduce costs and increase productivity. Two recent papers on the economic outcomes of breast cancer survivors suggest that use of health services is frequent and intensive during the first year ${ }^{52}$ and among long-term survivors whose overuse of medical resources for follow-up seems common..$^{53}$ Predictors of increased costs of services in the first year included comorbid illness, type of cancer treatment, depression, and physical function. Our finding that the negative impact of fatigue and pain, adjusted for all other factors, was greatest for the QOL dimension of role functioning suggests that addressing these symptoms may hasten return to usual activities, including work. Further research is needed to determine if more attention to the treatment of symptoms can reduce costs and unnecessary healthcare use. We also need to identify and target women disproportionately affected by breast cancer and its treatment, such as younger women and those with poor health status at the time of diagnosis, and offer more comprehensive interventions to address their unique needs and concerns.

\section{REFERENCES}

1. Rowland J, Mariotto A, Aziz NM, Tesauro G, Feuer EJ. Cancer survivorship-United States, 1971-2001. MMWR 2004;53:526.

2. U.S. Department of Health and Human Services. President's Cancer Panel. 2003-2004 annual report: Living beyond cancer: Finding a new balance. Available at deainfo.nci.nih.gov/ADVISORY/pcp/pcp.htm

3. Rustøen T, Begnum S. Quality of life in women with breast cancer: A review of the literature and implications for nursing practice. Cancer Nurs 2000;23:416.

4. Northouse LL, Templin T, Mood D. Couples' adjustment to breast disease during the first year following diagnosis. J Behav Med 2001;24:115.

5. Payne JK. The trajectory of fatigue in adult patients with breast and ovarian cancer receiving chemotherapy. Oncol Nurs Forum 2002;29:1334.

6. Bower JE, Ganz PA, Desmond KA, Rowland JH, Meyerowitz BE, Belin TR. Fatigue in breast cancer survivors: Occurrence, correlates, and impact on quality of life. J Clin Oncol 2000;18:743.

7. Smith WCS, Bourne D, Squair J, Phillips DO, Chambers A. A retrospective cohort study of post mastectomy pain syndrome. Pain 1999;83:91.

8. Beaulac SM, McNair LA, Scott TE, LaMorte WW, Kavanah MT. Lymphedema and quality of life in survivors of early-stage breast cancer. Arch Surg 2002; 137:1253.

9. Erickson VS, Pearson ML, Ganz PA, Adams J, Kahn K. Arm edema in breast cancer patients. I Natl Cancer Inst 2001;93:96.

10. Petrek JA, Heelan MC. Incidence of breast carcinomarelated lymphedema. Cancer 1998;83:276.

11. Carpenter JS, Andrykowski MA, Cordova M, et al. Hot flashes in postmenopausal women treated for breast carcinoma: Prevalence, severity, correlates, management, and relation to quality of life. $\underline{\text { Cancer }}$ 1998;82:1682.

12. Crandall C, Petersen L, Ganz PA, Greendale GA. Association of breast cancer and its therapy with menopause-related symptoms. Menopause 2004;11:519.

13. Ganz PA, Desmond KA, Belin TR, Meyerowitz BE, Rowland JH. Predictors of sexual health in women after a breast cancer diagnosis. I Clin Oncol 1999; 17:2371.

14. Stein K, Jacobsen P, Hann D, Greenberg H, Lyman G. Impact of hot flashes on quality of life among postmenopausal women being treated for breast cancer. I Pain Symptom Manage 2000;19:436.

15. Harris PF, Remington PL, Trentham-Dietz A, Allen CI, Newcomb PA. Prevalence and treatment of menopausal symptoms among breast cancer survivors. I Pain Symptom Manage 2002;23:501.

16. Davidson JR, MacLean AW, Brundage MD, Schulze K. Sleep disturbance in cancer patients. Soc Sci Med 2002;54:1309.

17. Savard J, Morin CM. Insomnia in the context of cancer: A review of a neglected problem. J Clin Oncol 2001;19:895. 
18. Fortner BV, Stepanski EJ, Wang SC, Kasprowicz S, Durrence HH. Sleep and quality of life in breast cancer patients. J Pain Symptom Manage 2002;24:471.

19. Shimozuma K, Ganz PA, Petersen L, Hirji K. Quality of life in the first year after breast cancer surgery: Rehabilitation needs and patterns of recovery. Breast Cancer Res Treat 1999;56:45.

20. Ganz PA, Greendale GA, Petersen L, et al. Breast cancer in younger women: Reproductive and late health effects of treatment. J Clin Oncol 2003;21:4184.

21. King MT, Kenny P, Shiell A, Hall J, Boyages J. Quality of life three months and one year after first treatment for early stage breast cancer: Influence of treatment and patient characteristics. Qual Life Res 2000;9:789.

22. Mandelblatt JS, Figueiredo MI, Cullen J. Outcomes and quality of life following breast cancer treatment in older women: When, why, how much, and what do women want? Health Qual Life Outcomes 2003;1:45.

23. Joly F, Espie M, Marty M, et al. Long-term quality of life in premenopausal women with node-negative localized breast cancer treated with or without adjuvant chemotherapy. Br J Cancer 2000;83:577.

24. Stanton AL, Ganz PA, Kwan L, et al. Outcomes from the Moving Beyond Cancer psychoeducational, randomized, controlled trial with breast cancer patients. J Clin Oncol 2005;23:6009.

25. Giedzinska AS, Meyerowitz BE, Ganz PA, Rowland $\mathrm{JH}$. Health-related quality of life in a multiethnic sample of breast cancer survivors. Ann Behav Med 2004; 28:39.

26. Ganz PA, Kwan L, Stanton AL, et al. Quality of life at the end of primary treatment of breast cancer: First results from the Moving Beyond Cancer randomized trial. J Natl Cancer Inst 2004;96:376.

27. Warner NJ, King M, Langlands AO, Kenny P, Boyages J. Symptoms 2 weeks, 3 months, and 12 months after treatment of early breast cancer: The patients' perspective. Breast 1999;8:273.

28. Arndt V, Stegmaier C, Ziegler H, Brenner H. A population-based study of the impact of specific symptoms on quality of life in women with breast cancer 1 year after diagnosis. Cancer 2006;107:2496.

29. Anema MG, Brown BE. Increasing survey responses using the total design method. J Contin Educ Nurs 1995;26:109.

30. Aaronson NK, Ahmedzai S, Bergman B, et al. The European Organization for Research and Treatment of Cancer QLQ-C30: A quality-of-life instrument for use in international clinical trials in oncology. J Natl Cancer Inst 1993;85:365.

31. Sprangers MA, Groenvold M, Arraras JI, et al. The European Organization for Research and Treatment of Cancer breast cancer-specific quality-of-life questionnaire module: First results from a three-country field study. J Clin Oncol 1996;14:2756.

32. McLachlan SA, Devins GM, Goodwin PJ. Factor analysis of the psychosocial items of the EORTC QLQC30 in metastatic breast cancer patients participating in a psychosocial intervention study. Qual Life Res 1999;8:311.

33. Niezgoda HE, Pater JL. A validation study of the domains of the core EORTC quality of life questionnaire. Qual Life Res 1993;2:319.

34. King MT. The interpretation of scores from the EORTC quality of life questionnaire QLQ-C30. Qual Life Res 1996;5:555.

35. Osoba D, Rodrigues G, Myles J, Zee B, Pater JL. Interpreting the significance of changes in health-related quality of life scores. I Clin Oncol 1998;16:139.

36. Fleming ID, Cooper JS, Henson DE, et al. American Joint Committee on Cancer staging manual. Philadelphia, PA: JB Lippincott, 1997.

37. Hartl K, Janni W, Kastner R, et al. Impact of medical and demographic factors on long-term quality of life and body image of breast cancer patients. Ann Oncol 2003;14:1064.

38. Jordhoy MS, Fayers P, Loge JH, Saltnes T, AhlnerElmqvist M, Kaasa S. Quality of life in advanced cancer patients: The impact of sociodemographic and medical characteristics. Br J Cancer 2001; 85:1478.

39. Mor V, Malin M, Allen S. Age differences in the psychosocial problems encountered by breast cancer patients. I Natl Cancer Inst Monogr 1994;16: 191.

40. Kroenke CH, Rosner B, Chen WY, Kawachi I, Colditz GA, Holmes MD. Functional impact of breast cancer by age at diagnosis. J Clin Oncol 2004;22:1849.

41. Avis NE, Crawford S, Manuel J. Quality of life among younger women with breast cancer. I Clin Oncol 2005;23:3322.

42. Baron R, Kelvin J, Bookbinder M, Cramer L, Borgen PI, Thaler HT. Patients' sensations after breast cancer surgery. Cancer Pract 2000;8:215.

43. Jung BF, Ahrendt GM, Oaklander AL, Dworkin RH. Neuropathic pain following breast cancer surgery: Proposed classification and research update. Pain 2003;104:1.

44. Baucom DH, Porter LS, Kirby JS, Gremore TM, Keefe FJ. Psychosocial issues confronting young women with breast cancer. Breast Dis 2005-2006;23:103.

45. Fobair P, Stewart SL, Chang S, D'Onofrio C, Banks PJ, Bloom JR. Body image and sexual problems in young women with breast cancer. Psychooncology 2006;15: 579.

46. Mols F, Vingerhoets A, Coebergh J, van de Poll-Franse L. Quality of life among long-term breast cancer survivors: A systematic review. Eur J Cancer 2005;41: 2613.

47. Schou I, Ekeberg O, Sandvik L, Hjermstad MJ, Ruland CM. Multiple predictors of health-related quality of life in early stage breast cancer: Data from a year follow-up study compared with the general population. Qual Life Res 2005;14:1813.

48. Perez DG, Loprinzi CL. Newer antidepressants and other nonhormonal agents for the treatment of hot flashes. Compr Ther 2005;31(3):224. 
49. Cella D, Kallich J, McDermott A, Xu X. The longitudinal relationship of hemoglobin, fatigue, and quality of life in anemic cancer patients: Results from five randomized clinical trials. Ann Oncol 2004;15:979.

50. Cimprich B. Development of an intervention to restore attention in cancer patients. Cancer Nurs 1993; 16:83.

51. Cimprich B, Ronis D. An environmental intervention to restore attention in women with newly diagnosed breast cancer. Cancer Nurs 2003;26:284.

52. Mandelblatt JS, Lawrence WF, Cullen JS, et al. Patterns of care in early-stage breast cancer survivors in the first year after cessation of active treatment. J Clin Oncol 2006;24:77.
53. Hensley ML, Dowell J, Herndon JE III, et al. Economic outcomes of breast cancer survivorship: CALGB study 79804. Breast Cancer Res Treat 2005;91:153.

Address reprint requests to: Nancy K. Janz, Ph.D.

Professor, Department of Health Behavior and Health Education University of Michigan, School of Public Health 109 Observatory Street Ann Arbor, MI 48109-2029

E-mail: nkjanz@umich.edu 


\section{This article has been cited by:}

1. Tsogzolmaa Dorjgochoo, Asha Kallianpur, Ying Zheng, Kai Gu, Zhi Chen, Wei Zheng, Wei Lu, Xiao Ou Shu. 2009. Impact of menopausal symptoms on quality of life 6 months after systemic breast cancer treatment: results from the Shanghai Breast Cancer Survival Study. Breast Cancer Research and Treatment . [CrossRef]

2. Rose C. Maly, Yihang Liu, Barbara Leake, Amardeep Thind, Allison L. Diamant. 2009. Treatment-related symptoms among underserved women with breast cancer: the impact of physician-patient communication. Breast Cancer Research and Treatment . [CrossRef]

3. Julie L. Otte, Janet S. Carpenter. 2009. Theories, Models, and Frameworks Related to Sleep-Wake Disturbances in the Context of Cancer. Cancer Nursing 32:2, 90-104. [CrossRef]

4. Fulya Balak, Corné A. M. Roelen, Petra C. Koopmans, Elike E. Berge, Johan W. Groothoff. 2008. Return to Work After Early-stage Breast Cancer: A Cohort Study into the Effects of Treatment and Cancer-related Symptoms. Journal of Occupational Rebabilitation 18:3, 267-272. [CrossRef] 